\title{
MicroED methodology and development
}

Brent L. Nannenga brent.nannenga@asu.edu

Microcrystal electron diffraction, or MicroED, is a method that is capable of determining structure from very small and thin 3D crystals using a transmission electron microscope. MicroED has been successfully used on microcrystalline samples including proteins, peptides and small organic molecules, in many cases to very high resolutions. In this presentation, the MicroED workflow will be described and areas of future methods development will be highlighted. These areas include improvements in sample preparation, data collection, and structure refinement. 